\title{
Effect of Superhelical Structure on the Secondary Structure of DNA Rings
}

\author{
DANIEL GLAUBIGER and JOHN E. HEARST, \\ Department of Chemistry, University of California, Berkeley, California
}

\section{Synopsis}

A quantity, called the linking number, is defined, which specifies the total number of twists in a circular helix. The linking number is invariant under continuous deformations of the ring and therefore enables one to calculate the influence of superhelical structures on the secondary helix of a circular molecule. The linking number can be determined by projecting the helix into a plane and counting strand crosses in the projection as described. For example, it has been shown that for each $180^{\circ}$ twist in a left-handed superhelix, a right-handed $360^{\circ}$ twist is removed from the secondary helix, thus allowing local unwinding.

\section{Introduction}

It is of interest to relate superhelical structure of helical molecules to their secondary structure. ${ }^{1}$ By defining a quantity characteristic of twostranded circular helical molecules, related to the helical structure, and invariant under continuous deformations of the molecule, we are able to evaluate the changes in helical structure brought about by the imposition of superhelical configurations on such molecules.

The quantity of interest, called the linking number, is related to the number of turns/cycle for a circular helical molecule.* It will be shown that superhelical structures which are made from helical polymers contribute to this number and alter the contribution made by the secondary structure of the molecule to this number. This is made manifest as a change in the number of turns/cycle or as a change in the number of turns/ unit length.

The linking number of a helix is defined as the number of $180^{\circ}$ revolutions about the helix axis required to reduce the helix to two disjoint strands. A reentrant helix has linking number equal to the number of twists per cycle where 1 cycle is the transversing of $2 \pi$ radians along the helix axis in the positive (counterclockwise) direction, starting from a fixed initial point. This amounts to choosing a starting point on the helix axis,

* It is also related, under an appropriate projection, to the winding number of the closed curve formed by the projected helix about the point corresponding to the helix axis. Winding number is defined as in Ahlfors, ${ }^{2}$ p. 93. 
and then following the contour in a counterclockwise direction until the chosen point is again reached. By convention, a right-handed helix has positive linking number.

\section{Calculation}

For a circular two-stranded helix, we let the helix axis lie in the $x y$ plane and let the center of the circle it forms be at the origin. We project the helix onto the $x y$ plane, maintaining the distinction between strands. Such a projection will show the strands intersecting each other at a certain number of points. At each intersection, we note which strand in the helix had the more positive $z$ value at that point prior to projection. We now traverse the projection completely in a counterclockwise direction along both strands, starting at any non-intersection point. The strand closer to the origin is the a strand. The other strand is the $\mathbf{b}$ strand.

With each intersection point of the projection we associate a number obtained in the following manner: If, at the intersection a "crosses" (is above) $\mathbf{b},+1$ is associated. If $\mathbf{b}$ crosses $\mathbf{a},-\mathbf{1}$ is associated. Now look at the tangents to $a$ and $b$ at the intersection point, denoted $t_{\mathbf{a}}$ and $t_{\mathbf{b}}$ (Fig. 1). If the angle between $t_{\mathbf{a}}$ and $t_{\mathbf{b}}$ is positive, where angles measured

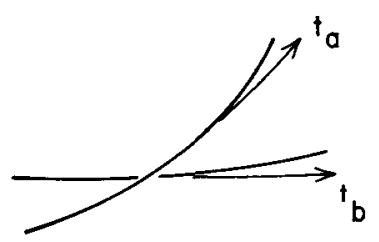

Fig. 1. Intersection of single strands resulting from the projection of a double stranded helix unto a plane. $t_{\mathrm{a}}$ and $t_{\mathrm{b}}$ represent the tangent vectors in the plane at the point of intersection. The break in strand $b$ signifies that $a$ passes over $\mathbf{b}$.

counterclockwise from $t_{\mathbf{a}}$ are positive, then +1 is associated with the interaction. If the angle between $t_{\mathbf{a}}$ and $t_{\mathbf{b}}$ is negative, -1 is associated. The product of these two parameters, one associated with the nature of the crossing, and the other with the angle of the crossing, is the number to be associated with the intersection. Thus, for example if a crosses $\mathbf{b}(+1)$ and the angle between $t_{\mathbf{a}}$ and $t_{\mathbf{b}}$ is negative $(-1),-1$ is associated with the intersection. This association is made with each intersection. The total of these numbers around 1 cycle (one complete traverse of the contour of the ring) is the linking number. As we have defined it, it is positive for right-handed helices and negative for left-handed helices. It is invariant under continuous deformation of the original helix, since an alteration in the linking number would require a discontinuity in the deformation.

What the linking number intuitively represents, for two-stranded circular helices, is the net number of clockwise $180^{\circ}$ rotations per cycle. (i.e., it measures the net number of right half turns about the helix axis per cycle.) 


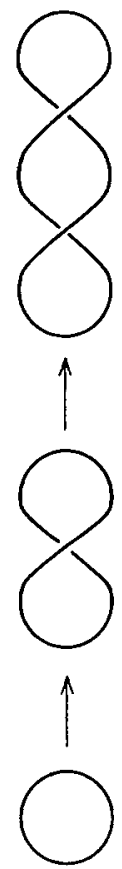

Fig. 2. Pictorial representation of a superhelix of zero, one, and two $180^{\circ}$ right-handed twists.

\section{Superhelical Structures}

The imposition of superhelical structures on a circular helix introduces intersections of order greater than 1 into the projection where more than two strands intersect. We will restrict our discussion to supercoils of first order (where the helix is itself wrapped into a helix, e.g., Fig. 2) for the present. If we consider a circular helix of linking number $+N$ which is itself wrapped into a right-handed helix as shown in Figure 2, then under an appropriate projection, the following situation pertains at the intersection of order 2 (Fig. 3).

Discounting $\mathbf{a}-\mathbf{a}$ and $\mathbf{b}-\mathbf{b}$ intersections we have $2 \mathbf{a}-\mathbf{b}$ intersections:

$\mathbf{a}$ crosses $\mathbf{b}$, the angle between $t_{\mathbf{a}}$, and $t_{\mathbf{b}}<0$

$$
(+1) \quad(-1) \quad=-1
$$

and

$\mathbf{b}$ crosses $\mathbf{a}$, the angle between $t_{\mathbf{a}}$ and $t_{\mathbf{b}}>0$

$$
(-1) \quad(+1) \quad=-1
$$

and the contribution to the linking number is -2 . However, since such a superhelical structure is obtainable by a continuous deformation of the original helix, the linking number is unchanged. Hence the contribution of this double intersection must be counterbalanced by single intersection 


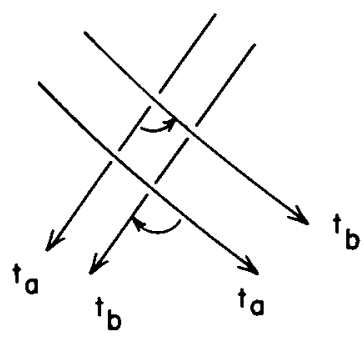

Fig. 3. Intersection of single strands in a projection plane at the crossings of Figure 2.

contributions totalling +2 . The argument extends to show that for each $180^{\circ}$ twist in a right-handed supercoil of the type described, two right half turns are introduced into the helix. The contributions are reversed for left-handed supercoils, and the number of right-handed half turns in the helix will be reduced by two for each $180^{\circ}$ twist of the supercoil.

Another first-order supercoil of interest is the reentrant torus shown in Figure 4. Under an appropriate projection, a right-hand torus will yield intersections as shown in Figure 5, which contribute

(b crosses a) and (the angle between $t_{\mathbf{a}}$ and $t_{\mathbf{b}}$ is negative)

$$
(-1) \times(-1) \quad=+1
$$

The contribution to the linking number is +1 unit per intersection. There are two such intersections per turn of the superhelical structure, and because of the reentrant boundary condition of the helix itself, there must be an integral number of full turns in the superhelix per cycle. Hence the contribution of the superhelix to the linking number per cycle of superhelix is an even integer.

Another structure of interest which is related to the torus we have discussed is shown in Figure 6. Discounting the loop made by reentrancy, each loop of the superhelix contributes tone full turn to the helical structure. The sign of the contribution is determined in the usual manner, one can readily verify that if the helix axis has the configuration of Figure

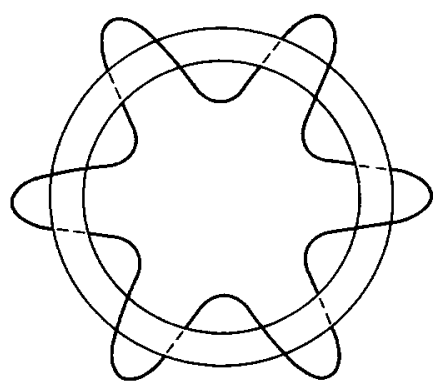

Fig. 4. The torus, another possible superhelix for a double-stranded DNA ring. 


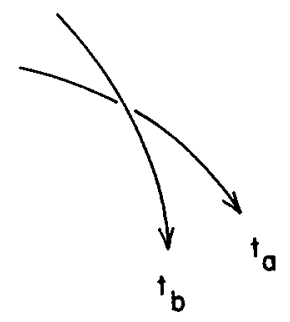

Fig. 5. Typical single strand crossing in a projection plane made necessary simply because of the torus geometry of Figure 4.

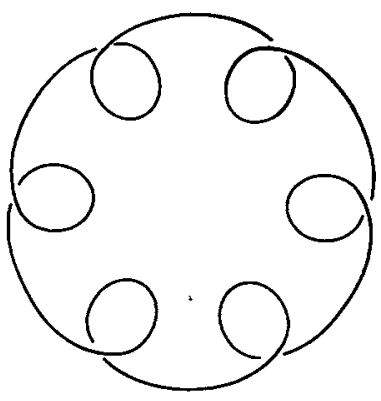

Fig. 6. Another possible superhelix.

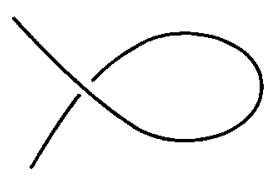

a

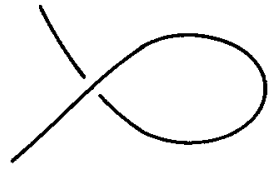

b

Fig. 7. Typical double-stranded loop resulting from the geometry of Figure 6.

$7 a$ the contribution is -2 , whereas if the configuration is that of Figure $7 b$ the contribution is +2 .

The net contribution of the superhelical structure to the linking number per cycle is the sum of the individual contributions of the loops contained in the cycle.

By considering the linking number of circular helical molecules we have shown that the imposition of superhelical structure on such a molecule will alter the number of turns/unit length of the original helix. The linking number remains invariant.

Hence by winding a helix into a supercoil one can alter substantially the properties of a helical molecule which depend on local interactions, or, alternatively, one could alter the percentage of the molecule involved in local interactions.

The authors wish to thank Professor J. Vinograd for introducing this problem to them. This work was supported in part by U. S. Public Health Service Grants No. GII-10840 and No. GM-11180. 


\section{References}

1. J. Vinograd, J. Lebowitz, R. Radloff, R. Watson, and P. Larkis, Proc. Natl. Acad. Sci. U.S., 53, 1104 (1965).

2. L. V. Ahlfors, Complex Analysis, McGraw-Hill, New York, 1953.

3. V. Bode and A. Kaiser, J. Mol. Biol., 14, 399 (1965).

4. A. Burton and R. Sinsheimer, J. Mol. Biol., 14, 327 (1965).

5. L. Crawford, J. Mol. Biol., 13, 362 (1965).

Received January 19, 1967

Prod. No. B310 